\title{
Collaborative practice among obstetricians, family physicians and midwives
}

\author{
Lisa Morgan RM MA(Midwifery practice), George Carson MD, Andrée Gagnon MD, Jennifer Blake MD MSc
}

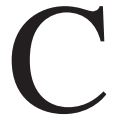
are during pregnancy and childbirth is provided by midwives, family practitioners and obstetrician-gynecologists, either individually or in collaboration. These groups have unique but often overlapping areas of competence and expertise. Those who provide such care are declining in number in this country. Canada currently has only 1650 obstetricians and gynecologists, not all of whom practise obstetrics, and about 600 plan to retire within the next five years. ${ }^{1}$ The country has just over 1000 midwives and, regrettably, a decreasing number of family physicians who provide maternity care - only 2142 based on the 2013 National Physician Survey. ${ }^{2}$ Of concern is that Canada lacks the ability to supply the maternity care, particularly intrapartum care, required in rural and remote, inner-city and Aboriginal communities. What is the solution?

A 2013 Cochrane review ${ }^{3}$ found that most outcomes of childbirth did not differ by type of health care provider. These outcomes included perineal trauma, induction and augmentation of labour, cesarean delivery, antenatal hospital admission, postpartum hemorrhage, length of hospital stay, initiation of breast feeding, neonatal Apgar scores, admission to neonatal nursery, and fetal loss after 24 weeks' gestation. ${ }^{3}$ Midwife-led care was associated with less use of analgesia and anesthesia, fewer episiotomies (with equivalent rates of intact perineum), fewer instrumental deliveries and a decrease in preterm births, but a longer length of labour. ${ }^{3}$ Other models of care were linked to an increase in interventions compared with midwife-led care, and a shorter time in labour. ${ }^{3}$ The review concluded that " $[\mathrm{m}]$ ost women should be offered midwife-led continuity models of care," while cautioning about those with substantial medical and obstetric complications. Does this mean that the answer to Canada's problem is to train more midwives?

Although that may be part of the solution, we are concerned that the Cochrane review appeared to go beyond the evidence in advocating for a midwife-led model of care in most cases. Although the review included 13 studies involving 16242 women, many of the studies are old and only one, from 1996, was a Canadian study, which enrolled only 218 women. Many of the included studies did not represent the kind of maternity care available in Canada, which also varies by province. The review garnered much media attention in Canada, ${ }^{4}$ which may not have been entirely useful in helping to improve public and professional understanding of what Canada needs to do to improve maternity services.

The effect of the human resources shortage on primary maternal and newborn care in Canada may be best attenuated by maximizing the interaction of health care providers through collaborative maternity care, which may also improve quality of care. ${ }^{5}$ We believe it is imperative that an inclusive Canadian strategy be established to support and promote appropriate collaborative models of maternity care that optimize and respect the skill set of each provider. This should be reflected at all stages of the organization and delivery of care, from initial visits to the postnatal period. Providing safe individualized care to each woman and newborn requires skilled care coordinated at the local, regional and provincial levels, ${ }^{1}$ and must also address the inequities in access, in particular for diverse and vulnerable populations.

Whereas an integrated system of delivering care would help to increase choice, provide highquality care and ensure good outcomes, there are several challenges to the development and delivery of collaborative maternity care in Canada. Resistance to change may be the biggest hurdle, because it is difficult to acknowledge that what we are currently doing is not providing the optimal results. ${ }^{6}$ In addition, most physicians and midwives are compensated under fee-for-service or course-of-care payment models, respectively,

\section{- KEY POINTS}

- Midwives, family practitioners and obstetrician-gynecologists have unique but overlapping areas of competence and expertise.

- Canada is lacking providers of maternity care, especially intrapartum care, in rural and remote, inner-city and Aboriginal communities.

- Efficient use of human resources requires maximizing the interaction of health care providers through collaborative maternity care.

- Providers of maternity care need to work together to create multidisciplinary, collaborative models of maternity and newborn care. 
which may be barriers to collaboration. ${ }^{6}$ Professionals are not necessarily prepared through education or socialization to work in the ways that characterize collaborative practice. ${ }^{7}$ Furthermore, regulatory and medicolegal issues can also complicate the relationship between maternity care providers and have made changing practice patterns difficult. Communication between health care providers also presents challenges when considering collaborative care models. Poor communication can result in poor team dynamics and delivery of suboptimal care.

However, there are precedents for collaborative maternity care. Both the Advances in Labour and Risk Management (ALARM) training program in emergency obstetrics (http://sogc.org /events/advances-in-labour-and-risk-management /welcome) and the Managing Obstetrical Risk Efficiently $\left(\mathrm{MORE}^{\mathrm{OB}}\right.$ ) program (www.moreob.com) are founded on interprofessional collaboration, communication and teamwork. These were developed jointly by obstetricians and family doctors under the auspices of The Society of Obstetricians and Gynaecologists of Canada (SOGC). In 2013, The College of Family Physicians of Canada voted to adopt ALARM as its preferred obstetrics training program. These courses teach all care providers to communicate effectively with mutual respect.

The model of collaborative care that we advocate is the shared-care model ${ }^{3}$ of responsibility for the organization and delivery of care. Canadian examples include the South Community Birth Program in Vancouver, where family physicians, midwives, community health nurses and doulas, with obstetric consultation as required, provide care in a community-based, culturally appropriate, woman-centred manner during pregnancy, birth and the newborn period. In Montréal, at La Maison Bleue, a shared care model serves disadvantaged women and families. The team includes midwives, family physicians, nurses, social workers and doulas. Similar

\section{Box 1: Priorities for a national birthing initiative}

1. Ensure that the needs and expectations of women during pregnancy and childbirth are met, regardless of where in Canada they live.

2. Facilitate engagement, collaboration and networking among professionals and government representatives involved in maternity and newborn care.

3. Establish a process for collection of data and information on maternity care providers and outcomes.

4. Create standardized clinical practice guidelines for all maternity care providers.

5. Adopt standardized curricula for maternity care education.

6. Establish interprofessional continuing education to manage risks, to improve patient safety and to facilitate collaborative woman-centred practice.

7. Establish multidisciplinary collaborative models for maternity and newborn care. models also exist in parts of Quebec, Ontario and other locations in Canada (Tonia Occhionero, Executive Director, Canadian Association of Midwives: personal communication, 2014).

Several national-level initiatives involving collaboration between maternity care providers currently exist. The Multidisciplinary Collaborative Primary Maternity Care Project aims to reduce barriers and facilitate the implementation of national, multidisciplinary, collaborative strategies to improve the availability and quality of maternity services. ${ }^{5}$ Importantly, the project team recommended that collaborative care teaching sites for undergraduate and postgraduate education be linked with continuing professional development programs to expose students and postgraduate trainees to these programs and to role models. ${ }^{5}$ Furthermore, The College of Family Physicians of Canada, the Canadian Association of Midwives, the Association of Women's Health, Obstetric and Neonatal Nurses, and the Society of Rural Physicians of Canada worked in cooperation with the SOGC to create A National Birthing Initiative for Canada. ${ }^{1}$

In this initiative, seven priorities for action are suggested (Box 1). Additional fora are needed to allow physicians, nurses, midwives and other maternity care providers an opportunity to discuss their respective challenges and successes with colleagues. ${ }^{5}$ Midwives, nurses, family physicians and obstetricians need to work together to create clinical practice guidelines and seek endorsement from other professional societies that represent areas where expertise and practice may overlap. Front-line leaders in the delivery of maternity care in Canada must work together to implement the national birthing initiative to ensure that Canadian women and their babies receive appropriate care during pregnancy, delivery and recovery. ${ }^{1}$ In our field, communication is central to good outcomes. The quality of our practice will improve if we work together from a collaborative model of clinical practice.

See references, www.cmaj.ca/lookup/suppl/doi:10.1503 /cmaj.140537/-/DC1

Affiliations: Laurentian University (Morgan), Sudbury, Ont.; Regina Qu'Appelle Health Region (Carson), Regina, Sask.; Hôpital régional de Saint-Jérôme (Gagnon), Saint-Jérôme, Que.; The Society of Obstetricians and Gynaecologists of Canada (Blake), Ottawa, Ont.

Contributors: Lisa Morgan conducted background research for the manuscript, was responsible for composing the first draft and was involved in revisions of subsequent drafts. George Carson, Jennifer Blake and Andrée Gagnon contributed substantially to the content of the manuscript, and assisted in its revisions. All of the authors approved the final version submitted for publication.

Acknowledgement: The authors acknowledge Becky Skidmore for assisting in background research, verifying references and coordinating the manuscript submission. 